\title{
Preliminary macroseismic survey of the 2016 Amatrice seismic sequence
}

\author{
MARIANO ANGELO ZANINI*, LORENZO HOFER, FLORA FALESCHINI, \\ PAOLO ZAMPIERI, NiCOLA FABRIS, CARLO PELLEGRINO \\ University of Padova, Dept. of Civil, Environmental and Ar- \\ chitectural Engineering, Padova, Italy \\ *marianoangelo.zanini@dicea.unipd.it
}

\begin{abstract}
After the recent destructive L'Aquila 2009 and Emilia-Romagna 2012 earthquakes, a sudden MW 6.0 seismic event hit Central Italy on August 24, 2016. A low population density characterizes the area but, due to its nighttime occurrence, about 300 victims were registered. This work presents the first preliminary results of a macroseismic survey conducted by teams of the University of Padova. Macroseismic intensities were assessed according to the European Macroseismic Scale (EMS98) for 180 sites.
\end{abstract}

\section{INTRODUCTION}

n August 24, 2016, at 3:36 local time (1:36 UTC), most of the inhabitants of central Italy were woken up by a $\mathrm{M}_{W}$ 6.0 earthquake that occurred at the boundaries of Lazio, Umbria, Marche and Abruzzo regions. The epicenter was located by the Istituto Nazionale di Geofisica e Vulcanologia (INGV) seismic network [ISIDe 2016] at $42.70^{\circ} \mathrm{N}$ and $13.24^{\circ} \mathrm{E}$, between the Municipalities of Accumoli and Amatrice, in the Lazio region. The Central Apennines sector is highly prone to seismic hazard: in recent years other devastating events occurred with epicenters located in a range of $30 \mathrm{~km}$ far from the actual one, like the April 6, 2009 ( $\mathrm{M}_{\mathrm{W}}$ 6.29) L'Aquila event and the 1997 Umbria seismic sequence, characterized by a $\mathrm{M}_{\mathrm{W}}$ 5.97 mainshock. According to the Parametric Catalogue of Italian Earthquakes (CPTI15) [Rovida et al. 2016], historical information reveals that the seismic activity in the Monti Sibillini area is frequent and in the past centuries was characterized by highly destructive events: from the eighteen century a mean annual rate of about 0.25 events with magnitude $\mathrm{M}_{\mathrm{w}}$ higher than 4.0 was observed. The first evidences of damage induced by earthquake occurrences in the area of Accumoli were related to the effects of the July 1627 Monti della Laga event $\left(\mathrm{M}_{\mathrm{W}} 5.3\right.$, $\mathrm{I}_{\mathrm{O}}$ 7-8 MCS) [Monachesi and Castelli 1992]. It was followed by the severe October 7, 1639 ( $\mathrm{M}_{\mathrm{W}}$ 6.21, Io 8-9 MCS) [Castelli 2013] Amatrice earthquake, that seemed very similar to the actual seismic scenario. The Accumoli area suffered extensive damages also after the most destructive January 14, $1703\left(\mathrm{M}_{\mathrm{W}}\right.$ 6.92 , Io $10 \mathrm{MCS}$ ), and was re-struck by the May 12, 1730 Valnerina event $\left(\mathrm{M}_{\mathrm{W}}\right.$ 6.04, Io 7 MCS) [Guidoboni et al. 2007]. After about 150 years these territories were hit again by the November 7, 1883 Monti della Laga earthquake ( $\mathrm{M}_{W} 5.10$, Io 7 MCS) and in the twentieth century by other significant events with Io MCS 7-8 in 1916, 1950 [Tertulliani et al. 2006] and 1979. In the days following the August 24 event, teams of the University of Padova organized a field survey of the damaged areas with the aim to develop an exhaustive macroseismic assessment of the 
earthquake scenario, according to the European Macroseismic Scale (EMS98) [Grünthal 1998]. The survey was continuously updated until September 6, 2016 to better define the damage effects induced by the mainshock event.

\section{SURVEY METHODOLOGY}

The teams surveyed 180 sites, assessing the intensity level on the basis of the structural damage suffered by the residential building stock and classifying it according to the European Macroseismic Scale (EMS98) [Grünthal 1998]. Industrial buildings were not taken into account due to their lack of representativeness for intensity assessment purposes in the region. Short interviews on the perception of the effects experienced by the inhabitants integrated damage data. A vulnerability class was identified for each analyzed building, and failures were classified according to the EMS98 damage grades. The most common residential building types in the area are: one-or-two-story old masonry buildings in stone and low quality mortar, with lack of connections (vulnerability class A and B); recently retrofitted masonry buildings (mostly renovated after the 1997 Umbria sequence, to which a vulnerability class $D$ was assigned); and two-story seismically designed reinforced concrete frame structures (vulnerability class C). Historical buildings like churches, castles and towers were considered for the intensity assessment purposes only in sites where the building stock was undamaged or slightly affected.

\section{INTENSITY ASSESSMENT}

Differently from the 2012 Emilia-Romagna seismic sequence, characterized by relevant aftershocks comparable to the May 20 mainshock, only one event was characterized by a magnitude $\mathrm{M}_{\mathrm{W}}$ greater than 5.0 , about one hour after the 1:36 UTC $\mathrm{M}_{\mathrm{W}} 6.0$ mainshock: for this reason, the intensity map can be reasonably viewed as the effects of the main event. Intensity values were defined on the basis of the damage grades detected on the different vulnerability classes observed in the analyzed villages. In some centers, difficulties in the intensity assessment were experienced mainly due to differences between damage levels observed in old centers and on recent reinforced concrete buildings: hence, teams surveyed such sites more times, to reduce subjectivity judgment. Figure 1 illustrates the intensity $\mathrm{I}_{\mathrm{EMS}}$ distribution over the struck territories and Table 1 lists $\mathrm{I}_{\mathrm{EMS}}$ values for each site surveyed. Major intensities were observed in Amatrice and Pescara del Tronto, which were mainly justified by a diffused level 5 of damage to several masonry buildings (Figure 2). Damages compatible with a $\mathrm{I}_{\text {EMS }} 8$ were detected in villages in an area within a radius of about $12 \mathrm{~km}$ far from the instrumental epicenter: in these cases, most of the vulnerability class $B$ residential masonry buildings suffered level 3 of damage. Some particular situations of very slight damage were observed close to the instrumental epicenter zone in the villages of Vezzano, Colle d'Arquata and Spelonga, probably due to beneficial site effects. The IEMS 6 area instead extended asymmetrically northwards, about $30 \mathrm{~km}$ from the instrumental epicenter, whereas southwards effects seemed significantly attenuated. Some difficulties were encountered in the intensity evaluation of damage in the southeastern towns of the Abruzzo region, since many buildings were still presenting damage caused by the 2009 L'Aquila sequence, and the relatively slight level of ground shaking induced in these areas by the $\mathrm{M}_{\mathrm{W}} 6.0$ mainshock, if compared to the epicentral zones. Another challenging issue was related to the assessment in the Umbria towns, since most of them were seismically retrofitted after 1997 sequence: here the critical issue 
ANNALS OF GEOPHYSICS, 59, Fast Track 5, 2016; DOI: 10.4401/ag-7172

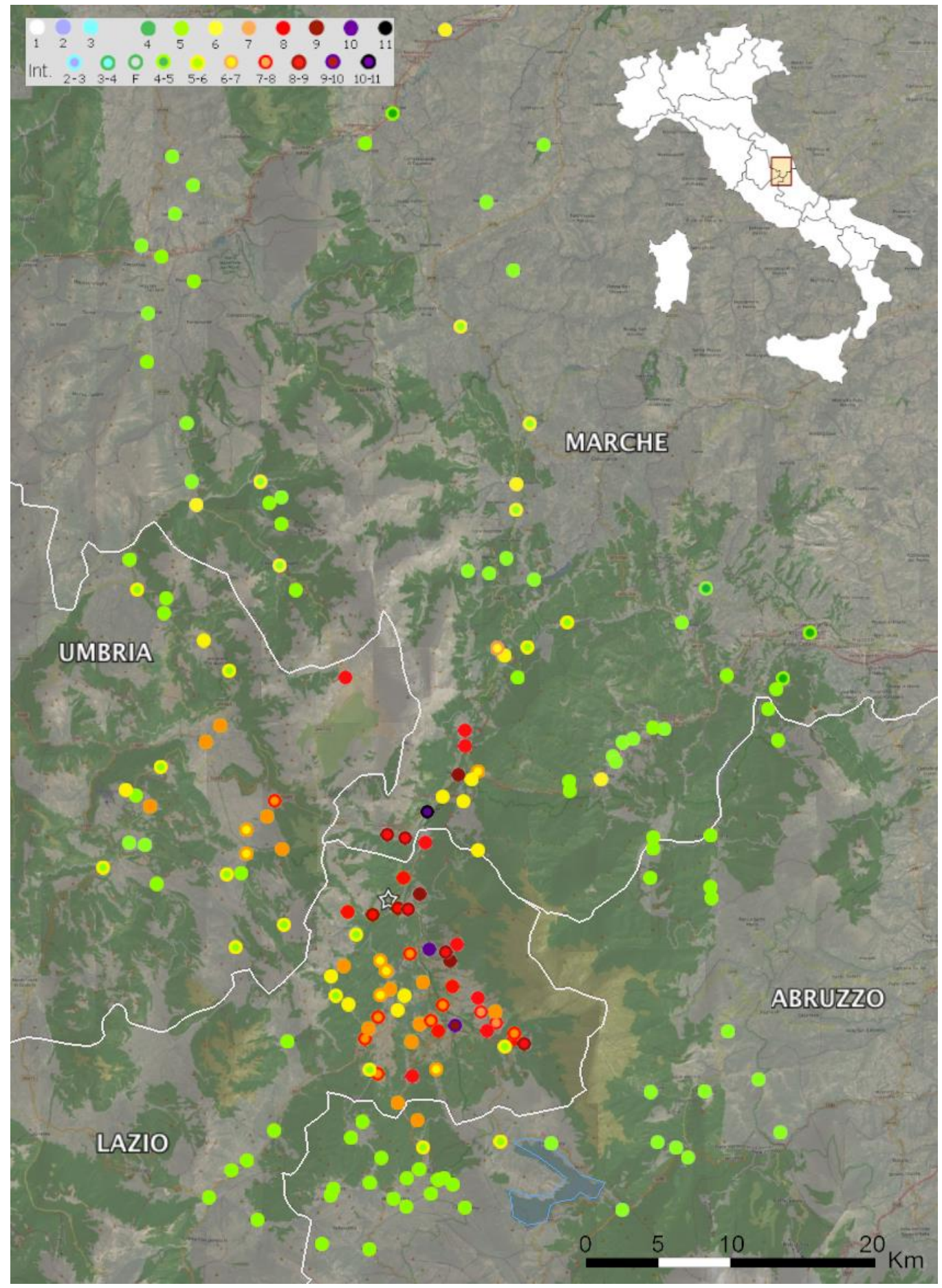

Figure 1: Macroseismic intensities observed after the August 2016 seismic sequence (damage up to September 6, 2016). 
ANNALS OF GEOPHYSICS, 59, Fast Track 5, 2016; DOI: 10.4401/ag-7172

Table 1: Localities surveyed after the August 2016 seismic sequence.

\begin{tabular}{|c|c|c|c|c|c|c|c|}
\hline $\mathbf{D}(*)$ & Municipality & Locality & $I_{\text {EMS }}$ & $\mathrm{D}(*)$ & Municipality & Locality & I $_{\text {EMS }}$ \\
\hline $\mathrm{AP}$ & Arquata del T. & Pescara del T. & $10-11$ & RI & Amatrice & Nommisci & $6-7$ \\
\hline RI & Amatrice & Saletta & 10 & PG & Norcia & Savelli & $6-7$ \\
\hline RI & Amatrice & Amatrice & $9-10$ & PG & Norcia & Valcadara & $6-7$ \\
\hline RI & Accumoli & Illica & 9 & $\mathrm{AP}$ & Arquata del T. & Trisungo & $6-7$ \\
\hline RI & Amatrice & San Lorenzo Fl. & 9 & RI & Accumoli & Roccasalli & 6 \\
\hline $\mathrm{AP}$ & Arquata del T. & Arquata del T. & 9 & RI & Amatrice & Pasciano & 6 \\
\hline RI & Accumoli & Accumoli & $8-9$ & RI & Amatrice & San Giorgio & 6 \\
\hline RI & Accumoli & Fonte del Campo & $8-9$ & RI & Amatrice & Santa Giusta & 6 \\
\hline RI & Accumoli & Villanova & $8-9$ & PG & Norcia & Agriano & 6 \\
\hline RI & Amatrice & Casale & $8-9$ & PG & Norcia & Campi & 6 \\
\hline RI & Amatrice & Preta & $8-9$ & $\mathrm{AP}$ & Acquasanta $\mathrm{T}$. & Acquasanta T. & 6 \\
\hline AP & Arquata del T. & Capodacqua & $8-9$ & $\mathrm{AP}$ & Arquata del T. & Colle & 6 \\
\hline $\mathrm{AP}$ & Arquata del T. & Tufo & $8-9$ & $\mathrm{AP}$ & Arquata del T. & Faete & 6 \\
\hline RI & Áccumoli & Grisciano & 8 & $\mathrm{AP}$ & Arquata del $\mathrm{T}$. & Spelonga & 6 \\
\hline RI & Accumoli & San Giovanni & 8 & $\mathrm{AP}$ & Arquata del T. & Vezzano & 6 \\
\hline RI & Accumoli & Tino & 8 & $\mathrm{AP}$ & Montegallo & Montegallo & 6 \\
\hline RI & Amatrice & Casteltrione & 8 & FM & Montefortino & Montefortino & 6 \\
\hline RI & Amatrice & Cornelle di Sotto & 8 & $\mathrm{MC}$ & Tolentino & Tolentino & 6 \\
\hline RI & Amatrice & Cossito & 8 & MC & Visso & Visso & 6 \\
\hline RI & Amatrice & Mosicchio & 8 & RI & Accumoli & Terracino & $5-6$ \\
\hline RI & Amatrice & Petrana & 8 & RI & Amatrice & Cornillo Nuovo & $5-6$ \\
\hline RI & Amatrice & Retrosi & 8 & RI & Amatrice & Forcelle & $5-6$ \\
\hline RI & Amatrice & Sommati & 8 & RI & Amatrice & Varoni & $5-6$ \\
\hline PG & Norcia & Castelluccio & 8 & PG & Cascia & Cascia & $5-6$ \\
\hline $\mathrm{AP}$ & Arquata del T. & Piedilama & 8 & PG & Cascia & Castel S. Maria & $5-6$ \\
\hline $\mathrm{AP}$ & Arquata del T. & Pretare & 8 & PG & Cascia & Civita & $5-6$ \\
\hline RI & Accumoli & Macchia & $7-8$ & PG & Norcia & Ospedaletto & $5-6$ \\
\hline RI & Amatrice & Bagnolo & $7-8$ & PG & Norcia & Pescia & $5-6$ \\
\hline RI & Amatrice & Capricchia & $7-8$ & PG & Norcia & Piè la rocca & $5-6$ \\
\hline RI & Amatrice & Collepagliuca & $7-8$ & PG & Preci & Preci & $5-6$ \\
\hline RI & Amatrice & Colli & $7-8$ & $\mathrm{AP}$ & Montegallo & Bisignano & $5-6$ \\
\hline RI & Amatrice & Cornillo Vecchio & $7-8$ & $\mathrm{AP}$ & Montegallo & Castro & $5-6$ \\
\hline RI & Amatrice & Moletano & $7-8$ & $\mathrm{AP}$ & Montegallo & Uscerno & $5-6$ \\
\hline RI & Amatrice & Scai & $7-8$ & FM & Amandola & Amandola & $5-6$ \\
\hline RI & Amatrice & Torrita & $7-8$ & FM & Montefortino & Cerretana & $5-6$ \\
\hline PG & Norcia & San Pellegrino & $7-8$ & MC & Castelsantangelo & Castelsantangelo & $5-6$ \\
\hline RI & Amatrice & Collegentilesco & 7 & MC & Sarnano & Sarnano & $5-6$ \\
\hline RI & Amatrice & Colleposta & 7 & MC & Ussita & Ussita & $5-6$ \\
\hline RI & Amatrice & Configno & 7 & $\mathrm{AQ}$ & Campotosto & Poggio Cancelli & $5-6$ \\
\hline RI & Amatrice & Patarico & 7 & $\mathrm{AQ}$ & Montereale & Aringo & $5-6$ \\
\hline RI & Amatrice & Poggio Vitellino & 7 & RI & Borbona & Borbona & 5 \\
\hline RI & Amatrice & Roccapassa & 7 & RI & Cittareale & Cittareale & 5 \\
\hline RI & Amatrice & San Benedetto & 7 & RI & Cittareale & Santa Croce & 5 \\
\hline RI & Amatrice & Voceto & 7 & RI & Posta & Bacugno & 5 \\
\hline PG & Cascia & Avendita & 7 & RI & Posta & Fontarello & 5 \\
\hline PG & Norcia & Fontevena & 7 & RI & Posta & Posta & 5 \\
\hline PG & Norcia & Frascano & 7 & PG & Norcia & Aliena & 5 \\
\hline PG & Norcia & Norcia & 7 & PG & Norcia & Fogliano & 5 \\
\hline PG & Norcia & Nottoria & 7 & PG & Norcia & Maltignano & 5 \\
\hline $\mathrm{AQ}$ & Montereale & Santa Lucia & 7 & PG & Norcia & Puro & 5 \\
\hline RI & Accumoli & Collespada & $6-7$ & PG & Norcia & Sant'Andrea & 5 \\
\hline RI & Amatrice & Collemoresco & $6-7$ & PG & Preci & Collescille & 5 \\
\hline RI & Amatrice & Domo & $6-7$ & PG & Preci & Piedivalle & 5 \\
\hline
\end{tabular}


ANNALS OF GEOPHYSICS, 59, Fast Track 5, 2016; DOI: 10.4401/ag-7172

\begin{tabular}{|c|c|c|c|c|c|c|c|}
\hline$D(*)$ & Municipality & Locality & $\mathrm{I}_{\mathrm{EMS}}$ & $\mathrm{D}(*)$ & Municipality & Locality & IEMS \\
\hline PG & Preci & Saccovescio & 5 & $\mathrm{AQ}$ & Capitignano & Capitignano & 5 \\
\hline $\mathrm{AP}$ & Ascoli Piceno & Castel Trosino & 5 & $\mathrm{AQ}$ & Capitignano & Collenoveri & 5 \\
\hline $\mathrm{AP}$ & Ascoli Piceno & Mozzano & 5 & $\mathrm{AQ}$ & Capitignano & Pago & 5 \\
\hline $\mathrm{AP}$ & Acquasanta $\mathrm{T}$. & Arli & 5 & $\mathrm{AQ}$ & Capitignano & Paterno & 5 \\
\hline $\mathrm{AP}$ & Acquasanta T. & Centrale & 5 & $\hat{A Q}$ & Capitignano & Sivignano & 5 \\
\hline $\mathrm{AP}$ & Acquasanta $\mathrm{T}$. & Corneto & 5 & $\mathrm{AQ}$ & Montereale & Casale Bottone & 5 \\
\hline $\mathrm{AP}$ & Acquasanta $\mathrm{T}$. & Novele & 5 & $\mathrm{AQ}$ & Montereale & Castiglione & 5 \\
\hline $\mathrm{AP}$ & Acquasanta $\mathrm{T}$. & Paggese & 5 & $\mathrm{AQ}$ & Montereale & Cesaproba & 5 \\
\hline $\mathrm{AP}$ & Acquasanta $\mathrm{T}$. & Ponte d'Arli & 5 & $\mathrm{AQ}$ & Montereale & Cesariano & 5 \\
\hline $\mathrm{AP}$ & Acquasanta $\mathrm{T}$. & Quintodecimo & 5 & $A Q$ & Montereale & Lonaro & 5 \\
\hline $\mathrm{AP}$ & Acquasanta $\mathrm{T}$. & San Martino & 5 & $\mathrm{AQ}$ & Montereale & Marana & 5 \\
\hline $\mathrm{AP}$ & Acquasanta $\mathrm{T}$. & Santa Maria & 5 & $\mathrm{AQ}$ & Montereale & Montereale & 5 \\
\hline $\mathrm{AP}$ & Montegallo & Forca & 5 & $\mathrm{AQ}$ & Montereale & Piedicolle & 5 \\
\hline $\mathrm{AP}$ & Montemonaco & Montemonaco & 5 & AQ & Montereale & San Vito & 5 \\
\hline $\mathrm{AP}$ & Montemonaco & Pignotti & 5 & $\mathrm{AQ}$ & Montereale & Santa Vittoria & 5 \\
\hline $\mathrm{AP}$ & Montemonaco & Rocca & 5 & $\mathrm{AQ}$ & Montereale & Verrico & 5 \\
\hline $\mathrm{AP}$ & Montemonaco & San Giorgio Isola & 5 & AQ & Montereale & Ville & 5 \\
\hline $\mathrm{AP}$ & Roccafluvione & Roccafluvione & 5 & $\mathrm{TE}$ & Cortino & Cortino & 5 \\
\hline $\mathrm{MC}$ & Caldarola & Caldarola & 5 & TE & Crognaleto & Alvi & 5 \\
\hline MC & Camerino & Camerino & 5 & $\mathrm{TE}$ & Crognaleto & Cesacastina & 5 \\
\hline MC & Camerino & San Luca & 5 & $\mathrm{TE}$ & Crognaleto & Crognaleto & 5 \\
\hline MC & Camerino & San Marcello & 5 & TE & Crognaleto & Nerito & 5 \\
\hline MC & Castelsantangelo & Gualdo & 5 & $\mathrm{TE}$ & Crognaleto & Poggio U. & 5 \\
\hline MC & Gualdo & Gualdo & 5 & TE & Crognaleto & San Giorgio & 5 \\
\hline $\mathrm{MC}$ & Muccia & Muccia & 5 & TE & Crognaleto & Tottea & 5 \\
\hline $\mathrm{MC}$ & Pieve Torina & Apennino & 5 & TE & Valle Castellana & Ceraso & 5 \\
\hline $\mathrm{MC}$ & Pieve Torina & Capriglia & 5 & TE & Valle Castellana & Cerquito & 5 \\
\hline $\mathrm{MC}$ & Pieve Torina & Pieve Torina & 5 & $\mathrm{TE}$ & Valle Castellana & Morrice & 5 \\
\hline MC & Pievebovigliana & Pievebovigliana & 5 & $\mathrm{TE}$ & Valle Castellana & Pascellata & 5 \\
\hline MC & Ripe S. Ginesio & Ripe S. Ginesio & 5 & $\mathrm{TE}$ & Valle Castellana & Pietralta & 5 \\
\hline MC & San Ginesio & San Ginesio & 5 & $\mathrm{TE}$ & Valle Castellana & San Vito & 5 \\
\hline MC & Ussita & Calcara & 5 & $\mathrm{TE}$ & Valle Castellana & Valle Castellana & 5 \\
\hline MC & Ussita & Cuore di Sorbo & 5 & $\mathrm{AP}$ & Ascoli Piceno & Ascoli Piceno & $4-5$ \\
\hline MC & Ussita & Frontignano & 5 & $\mathrm{AP}$ & Ascoli Piceno & Casette & $4-5$ \\
\hline MC & Visso & Borgo S. Antonio & 5 & $\mathrm{AP}$ & Venarotta & Venarotta & $4-5$ \\
\hline $\mathrm{AQ}$ & Campotosto & Campotosto & 5 & MC & Belforte & Belforte & $4-5$ \\
\hline $\mathrm{AQ}$ & Campotosto & Ortolano & 5 & MC & Muccia & Maddalena & $4-5$ \\
\hline
\end{tabular}

(*) Districts: Rieti (RI), Perugia (PG), Ascoli Piceno (AP), Fermo (FM), Macerata (MC), Aquila (AQ), Teramo (TE).

was to define the most reliable EMS98 vulnerability class, for properly derive the IEMS degree. A review of the local strengthening techniques suggested to adopt, in such cases, a vulnerability class D for masonry structures. Unreinforced buildings, if present, were also considered in these localities to check the reliability of the assigned intensity value.

\section{CONCLUSIONS}

Results of a macroseismic survey performed in the area struck by the August 24, 2016 Amatrice sequence were presented. A total number of 180 sites were surveyed and for each of them a IEMS value was defined. According to the results shown in this work, it can be concluded that site effects 

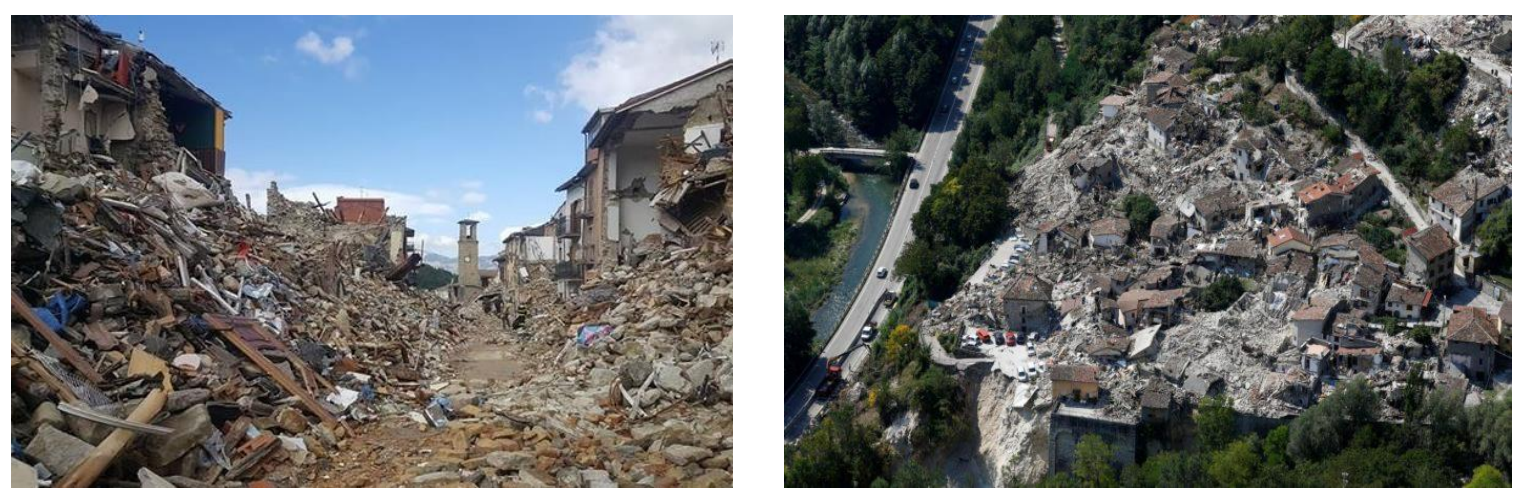

Figure 2: Diffused collapses in Amatrice (left) and aerial view of Pescara del Tronto (right, retrieved on www.meteoweb.eu).

significantly influenced the 2016 Central Italy sequence. Additionally, a key aspect of this work related the evaluation of the effects induced by the analyzed earthquake to predamaged and seismically retrofitted residential buildings, for a proper derivation of the a $\mathrm{I}_{\mathrm{EMS}}$ value. Finally, a preliminary analysis based on statistical inference of the EMS intensities assessed was performed, evidencing how the macroseismic epicenter seems located $4 \mathrm{~km}$ southeastwards with respect to the instrumental one.

\section{REFERENCES}

Castelli, V. (2013). Revisione delle conoscenze sui terremoti del 1558 (Valdambra), 1561 (Campania-Basilicata), 1639 (Amatriciano) e 1747 (Nocera Umbra Gualdo Tadino). Rapporto tecnico INGV-MI, Milano, 16 pp.

Grünthal, G., ed. (1998). European Macroseismic Scale 1998 (EMS-98). European Seismological Commission, Subcommission on Engineering Seismology, Working Group Macroseismic Scales. Conseil de l'Europe, Cahiers du Centre Europèen de Gèodynami- que et de Sèismologie, 15, Luxembourg, 99 pp.

Guidoboni, E., G. Ferrari, D. Mariotti, A. Comastri, G. Tarabusi and G. Valensise (2007) CFTI4Med, Catalogue of Strong Earthquakes in Italy (461 B.C. - 1997) and Meditteranean Area (760 B.C. - 1500). INGVSGA. http://storing.ingv.it/cfti4med.

ISIDe Working Group (INGV) (2016). Italian seismological instrumental and parametric database: http://iside.rm.ingv.it, DOI: 10.13127/ISIDe, last accessed September 2016.

Rovida, A., M. Locati, R. Camassi, B. Lolli, P. Gasparini, eds. (2016). CPTI15, the 2015 version of the Parametric Catalogue of Italian Earthquakes. Istituto Nazionale di Geofisica e Vulcanologia. doi:http://doi.org/ 10.6092/ INGV.IT-CPTI15.

Tertulliani, A., F. Galadini, F. Mastino, A. Rossi and M. Vecchi (2006) Studio macrosismico del terremoto del Gran Sasso (Italia Centrale) del 5 settembre 1950: implicazioni sismotettoniche. Il Quaternario, 19, 2: 195-214. 